\title{
Large Electron Transfer Rate Effects from the Duschinsky Mixing of Vibrations
}

\author{
Gerald M. Sando, Kenneth G. Spears,* and Joseph T. Hupp \\ Northwestern University, Department of Chemistry, 2145 Sheridan Road, Evanston, Illinois 60208

\begin{abstract}
Peder Thusgaard Ruhoff
Maersk Mc-Kinney Moller Institute for Production Technology, University of Southern Denmark, Odense University, Campusvej 55, DK-5230 Odense M, Denmark
\end{abstract}

Received: November 17, 2000; In Final Form: March 19, 2001

\begin{abstract}
We systematically test how the Duschinsky mixing of normal coordinate vibrations affects transition rates for electron transfer (ET). We find that ET rates in the inverted region can increase many orders of magnitude from Duschinsky mixing, and both totally symmetric and nontotally symmetric vibrations are very important. The Duschinsky effect arises when two electronic states have vibrational normal mode coordinate systems that are rotated and translated relative to each other. We use a conventional quantum rate model for ET, and the examples include 6-8 vibrations, where two vibrational modes are mixed with different amounts of coordinate rotation. The multidimensional Franck-Condon factors (FCF) are computed with standard algorithms and recently developed recursion relations. When displaced, totally symmetric modes are involved, rates with Duschinsky mixing can increase several orders of magnitude for inverted electron transfer reactions and modest mixing. The peak location in a rate vs energy gap plot can depend on the degree of Duschinsky mixing, and therefore it corresponds to a sum of solvent and an effective vibrational reorganization energy that is not predictable by simple models that exclude mixing. In addition, for some examples of inverted region ET we observe significant flattening of the usual parabolic curve at large degrees of mixing. We demonstrate that large rate effects can occur with very little change in either the calculated absorption or emission spectra, depending on the details of the Duschinsky mixing. The origin of the rate effect is the increased FCF between the initial vibrational state and the higher lying final vibrational states when the Duschinsky effect is taken into account. The rate effect of totally symmetric modes is greater than nontotally symmetric modes, but since there are many nontotally symmetric modes, in real molecules these modes can make a large total contribution to ET rates.
\end{abstract}

\section{Introduction}

Electron transfer (ET) reactivity has been the subject of enormous theoretical attention due to its ubiquity in chemistry and biology. ${ }^{1}$ However, one issue, the Duschinsky effect, ${ }^{2}$ has generally been ignored in almost all of the theoretical treatments. In this work we will show that there are many instances in which inclusion of the Duschinsky effect can lead to large changes in calculated electron-transfer rates. The theoretical analysis of the Duschinsky effect is described elsewhere. ${ }^{3,4}$ Basically, the Duschinsky effect describes the fact that upon a change in electronic state, the nature of the vibrational normal modes changes. The two sets of normal modes are approximately related by the linear transformation in eq $1 .^{2-5}$

$$
Q^{\prime}=J Q+K
$$

In eq $1, Q$ ' represents the normal coordinate vector of the initial state, $Q$ represents the normal coordinate vector of the final state, $J$ is the Duschinsky matrix, and $K$ is the vector of normal coordinate displacements between the equilibrium geometries of the two states. As described in another manuscript, ${ }^{6}$ the transformation is in general not linear unless certain conditions are true.

\footnotetext{
*E-mail: spears@chem.nwu.edu. Fax: (847) 491-7713.
}

As a result, when the effect is significant, full multidimensional Franck-Condon factors (FCF), rather than simple products of one-dimensional FCF, must be calculated. In developing the work described here, we have taken advantage of prior work by many authors on the inclusion of Duschinsky phenomenon in absorption ${ }^{7-14}$ and resonance Raman ${ }^{15-17}$ spectral simulation. However, in only a very few cases has the Duschinsky effect been included in calculations of nonradiative decay ${ }^{18,19}$ and ET ${ }^{20,21}$ rates. In the ET studies, the Duschinsky effect was for specific systems and had little effect upon the ET rate. The suggestion that Duschinsky effects might be important in ET has been made in earlier work from this laboratory, ${ }^{22}$ but no models have been available to describe the general role of the Duschinsky effect in ET rate processes, and this manuscript is the first systematic study. Our goal is to systematically study the Duschinsky effect on ET rates to determine when the Duschinsky effect can become important. Rather than using specific molecules, we have identified a simple model system with parameters that allow systematic changes while providing the properties of real molecules. To provide a familiar framework in terms of FCF, we use a recent development ${ }^{3}$ of recursion relations to compute Duschinsky kinetic effects.

One common result of the Duschinsky effect is the loss of mirror symmetry between otherwise electronically equivalent 
absorption and emission spectra, a point that has been well appreciated in several experimental studies. ${ }^{23-26}$ Underlying the phenomenological spectral symmetry loss is a more fundamental difference between ground state and excited state symmetry inherent to Duschinsky rotation of vibrational modes. One immediate consequence is that the numerical values of familiar and seemingly simple parameters, such as displacements and reorganization energies, become dependent upon which electronic state is used as a basis. Consequently, some care is required in defining and interpreting these parameters. At a more fundamental level, the asymmetry inherent to Duschinsky rotation implies that the typical interpretation of experimental ET rates versus free energy can be subject to misinterpretation of reorganization energy and curvature. In addition, we show many orders of magnitude enhancement of ET rate constants in the inverted region.

Finally, to avoid complications due to nonlinear or nonorthogonal transformations $\mathrm{s}^{3,4}$ that occur when axis-switching effects $^{27}$ are taken into account, and to permit the use of published methods ${ }^{5,28-33}$ for calculating FCF, some important constraints and assumptions have been introduced. Most importantly, to use a linear transformation of eq 1 we assume high symmetry point groups (not $C_{1}, C_{s}, C_{i}, C_{n}, C_{n h}$, or $S_{n}$ ). Additionally, when nontotally symmetric modes are considered we assume that they do not belong to the same representation as a rotation. Although these conditions and assumptions are satisfied in the model systems we have investigated, their applicability clearly must be examined before applying the analysis, without modification, to other ET systems.

\section{Methods}

To carry out systematic studies of the Duschinsky effect upon ET kinetics, the effect was limited to the mixing of just two of the 6-8 vibrational modes that are used in the model. For real molecules, one expects more than two modes to be mixed, but this allows simpler analysis of the effects without loss of generality. Under these conditions the Duschinsky matrix, $J$, becomes a simple two-dimensional rotation matrix, thereby facilitating the presentation of results as a function of one mixing parameter, the angle of rotation. This greatly eases visualization of the dependence of electron transfer rate on the amount of Duschinsky mixing. In the more general multidimensional case, many angles are needed to characterize the Duschinsky matrix; this not only renders the parameter space extremely large but also complicates data presentation. Parameters are chosen to be realistic for actual ET systems, although they are not based on any particular molecule. Realistic values of mixing depend on the molecule; but for comparison, an actual two-dimensional case results when considering the totally symmetric modes of $\mathrm{NO}_{2}$. Calculations of the ${ }^{2} \mathrm{~A}_{1}$ ground and ${ }^{2} \mathrm{~B}_{2}$ excited states at the UBP86/6-31G* level result in an NO bond length increase of $0.015 \AA$ and a bond angle decrease of $32^{\circ}$ in the excited state. Calculation of the Duschinsky effect results in a mixing angle of $18^{\circ}$ for the totally symmetric bend and stretch modes that have frequencies of 722.6 and $1333.5 \mathrm{~cm}^{-1}$ in the ${ }^{2} \mathrm{~A}_{1}$ state.

Electron-transfer rates were calculated based on a standard sum-over-states golden rule formulation: ${ }^{34,35}$

$$
\begin{aligned}
k_{\mathrm{ET}}=\frac{2 \pi}{\hbar} H_{\mathrm{ab}}^{2}\left(4 \pi \lambda_{2} k_{\mathrm{B}} T\right)^{-1 / 2} & \times \sum_{\mathrm{i}} P\left(\epsilon_{\mathrm{i}}\right) \sum_{\mathrm{f}}\left\langle\chi_{\mathrm{i}} \mid \chi_{\mathrm{f}}\right\rangle^{2} \\
& \exp \left[\frac{-\left(-E_{00}+\epsilon_{\mathrm{f}}-\epsilon_{\mathrm{i}}+\lambda_{\mathrm{s}}\right)^{2}}{4 \lambda_{\mathrm{s}} k_{\mathrm{B}} t}\right]
\end{aligned}
$$

In eq $2, \mathrm{i}$ is the initial (excited) state, $\mathrm{f}$ is the final (ground) state, $H_{\mathrm{ab}}$ is the electronic coupling of the two states, $\lambda_{\mathrm{s}}$ is the solvent reorganization energy, $E_{00}$ is the free energy difference, $\chi$ is the vibrational wave function, and $P\left(\epsilon_{\mathrm{i}}\right)$ is the distribution of initial vibrational states.

Electronic spectra were calculated at the same level of theory with the following equations, with the identities of the initial and final states reversed for absorption versus emission spectra. ${ }^{34}$

$$
\begin{gathered}
A(\omega) \propto \omega \sum_{\mathrm{i}} P\left(\epsilon_{\mathrm{i}}\right) \sum_{\mathrm{f}}\left\langle\chi_{\mathrm{i}} \mid \chi_{\mathrm{f}}\right\rangle^{2} \exp \left[\frac{-\left(\omega-E_{00}-\epsilon_{\mathrm{f}}+\epsilon_{\mathrm{i}}-\lambda_{\mathrm{s}}\right)^{2}}{4 \lambda_{\mathrm{s}} k_{\mathrm{B}} T}\right] \\
E(\omega) \propto \omega^{3} \sum_{\mathrm{i}} P\left(\epsilon_{\mathrm{i}}\right) \sum_{\mathrm{f}}\left\langle\chi_{\mathrm{i}} \mid \chi_{\mathrm{f}}\right\rangle^{2} \\
\exp \left[\frac{-\left(\omega-E_{00}+\epsilon_{\mathrm{f}}-\epsilon_{\mathrm{i}}+\lambda_{\mathrm{s}}\right)^{2}}{4 \lambda_{\mathrm{s}} k_{\mathrm{B}} T}\right]
\end{gathered}
$$

In view of computer time and memory limitation, the initial state was generally assumed to exist in the lowest vibrational level. In a few calculations, however, a thermal Boltzmann population was introduced, and we found little difference. The Franck-Condon factors were calculated by using recursion relations derived by Ruhoff. ${ }^{28}$ For the recursion algorithm used here, memory storage of all FCF was necessary; the implied memory storage problems proved most significant in calculations involving large energy gaps. The FCF algorithm was implemented in a FORTRAN code originally developed by Dr. Ruhoff, but modified at Northwestern to incorporate the summation in the rate, absorption, and emission equations.

Because we are using a sum-over-states method, we are limited in the number of modes we can consider in a calculation due to the rapid increase in terms with additional modes. While we have successfully performed rate calculations with up to 12 normal modes with reasonable energy gaps and solvent reorganization energies, this necessitated care in setting limits for the summation to reach convergence. In the calculations performed here, the 6 mode calculations took at most a few seconds on a $450 \mathrm{MHz} \mathrm{PC}$, while the 8 mode calculations required from a few seconds at small energy gaps to a few minutes at the largest energy gaps studied. It should be noted that the Duschinsky effect has been included in time dependent correlation function methods ${ }^{36-38}$ that can be applied to larger systems. $^{39}$

\section{Results and Discussion}

A. Totally Symmetric Modes-Basic ET Effects. Duschinsky Mixed Modes As Majority Acceptors: Case 1, Rotation of Ground State. A plot of electron-transfer rate as a function of energy gap is given in Figure 1, with the corresponding parameters in Table 1. In the limit of no mixing, the vibrational reorganization energy, $\lambda_{\mathrm{v}}$, is $2210 \mathrm{~cm}^{-1}$, with the dominant portion $(97 \%)$ coming from the two modes that are to be mixed, 500 and $1400 \mathrm{~cm}^{-1}$. The total reorganization energy, $\lambda_{\text {total }}$, is $5210 \mathrm{~cm}^{-1}$, defined as the sum of vibrational and solvent reorganization energy. When mixing is introduced, the rate in the inverted region $\left(E_{00}>\lambda_{\text {total }}\right)$ is greatly increased and we observe significant flattening of the usual parabolic curve at large degrees of mixing. An additional effect is that the maximum rate is no longer at an energy gap equal to the total reorganization energy (with no mixing), but shifts to higher energy with increased mixing. We interpret this shift as representing the effective reorganization energy. In Figure 1 


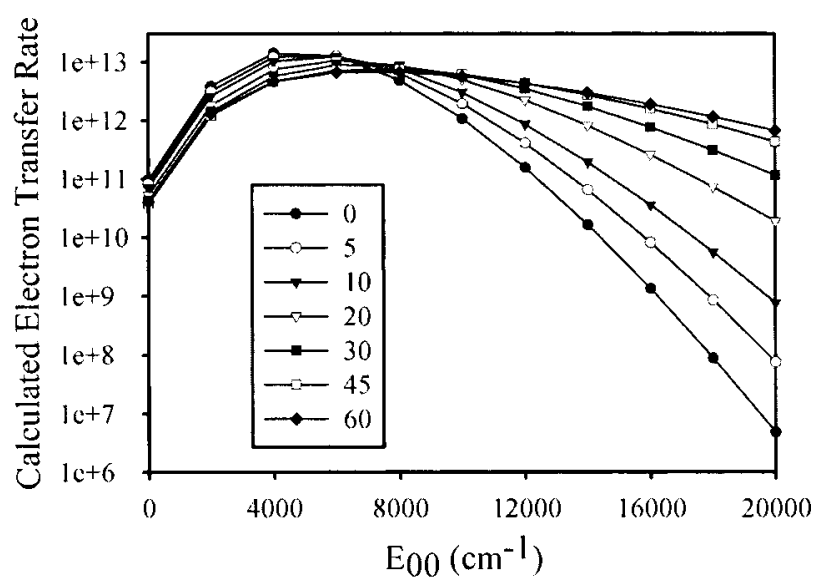

Figure 1. Calculated electron transfer rate vs energy gap for several values of mixing angle in Case 1, with a rotation of ground state normal coordinates. Parameters are given in Table 1, and only the $500 \mathrm{~cm}^{-1}$ and $1400 \mathrm{~cm}^{-1}$ modes undergo the Duschinsky effect while also serving to accept $97 \%$ of the reorganization energy.

TABLE 1: Parameters Used for the Calculations in Figure $\mathbf{1}^{a}$

\begin{tabular}{rclr}
\hline$v\left(\mathrm{~cm}^{-1}\right)$ & $\mathrm{K}\left(\mathrm{amu}^{0.5} \mathrm{\AA}\right)$ & $\Delta$ & $\lambda_{\mathrm{i}}\left(\mathrm{cm}^{-1}\right)$ \\
\hline 1524 & 0.03 & 0.2 & 31 \\
300 & 0.05 & 0.15 & 3 \\
1200 & 0.03 & 0.18 & 19 \\
3100 & 0.01 & 0.1 & 14 \\
$* 500^{b}$ & 0.39 & 1.5 & 564 \\
$* 1400^{b}$ & 0.233 & 1.5 & 1578
\end{tabular}

${ }^{a} \mathrm{H}_{\mathrm{ab}}=250 \mathrm{~cm}^{-1} ; \lambda_{\mathrm{s}}=3000 \mathrm{~cm}^{-1} ; \lambda_{\mathrm{v}}=\sum \lambda_{\mathrm{i}}=2209.7 \mathrm{~cm}^{-1} ; \lambda_{\mathrm{i}}=$ $0.5 v_{\mathrm{i}} \Delta_{\mathrm{i}} 2 ; \Delta_{\mathrm{i}}=0.17222 v_{\mathrm{i}} 0.5 K_{\mathrm{i}} .{ }^{b}$ Mixed modes.

the vibrational reorganization energy is described in groundstate coordinates, and the vibrational reorganization energy increases greatly, from $2210 \mathrm{~cm}^{-1}$ at no mixing to $9060 \mathrm{~cm}^{-1}$ at a $60^{\circ}$ mixing angle. This is in contrast to the usual assumption that the maximum rate occurs when the energy gap is equal to the total reorganization energy in a non-Duschinsky model. The reason for the energy shift in the maximum rate is that, for this case, the effective reorganization energy depends on the rotation angle of the ground-state coordinate system. This is dependent on the specific coordinate rotation, and examples where the maximum rate is independent of rotation angle are also possible. We review subtleties of coordinate rotation and translation in the next section.

Duschinsky Rotation and Reorganization Energy in ET Models. First we review some concepts of coordinate system rotation and how they affect concepts often used in discussing ET and optical absorption and emission. In the absence of the Duschinsky effect, the normal modes of each state involved in a transition can have relative displacements of the coordinate system, but no rotation of the coordinate system. Figure 2 has three cases for discussion, and the top drawing has a case for two modes drawn as elliptical energy contours showing energy versus displacement; these two states could have been drawn with different frequencies, but for simplicity they are identical. For example, one might imagine that the long axis of the ellipse is a lower frequency bend mode and the short axis is a higher frequency stretch mode. The top drawing shows the normal mode coordinate systems of the two states with no relative rotation. The displacements (dimensionless displacements, $\Delta$, or normal coordinate displacements, $\mathrm{K}$, with units of mass ${ }^{0.5}$ length) are a measure of how far along a particular normal mode the molecule in one state must move in order to reach the geometry of the other state. If a one-dimensional slice of the
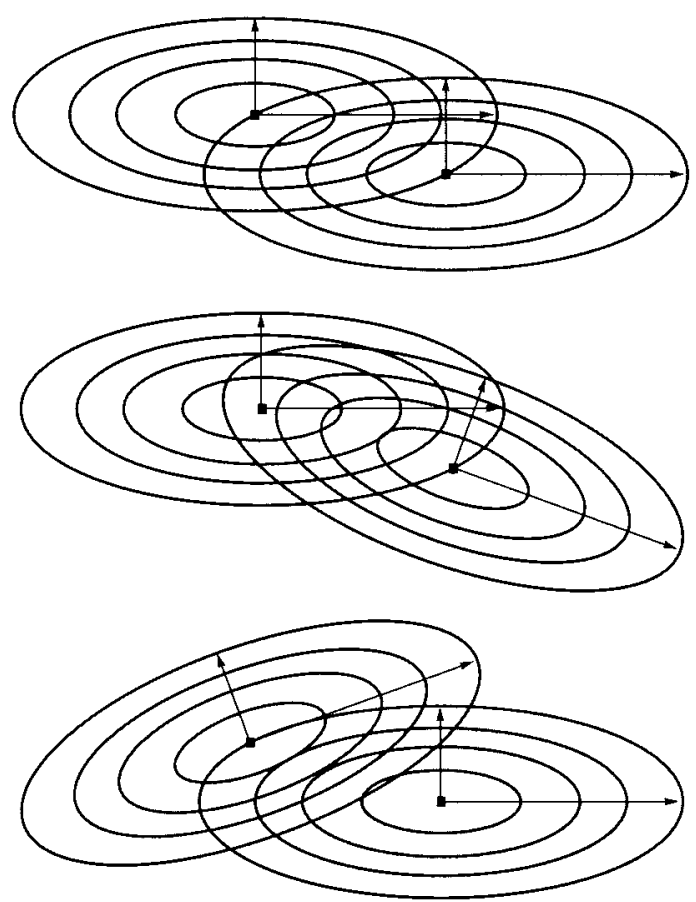

Figure 2. Potential energy surfaces for two normal coordinates in two different states. The excited state is the upper left state. The top drawing is the nonrotated case, the middle drawing has the ground-state coordinates rotated by $+20^{\circ}$, and the bottom drawing has the excitedstate rotated by $-20^{\circ}$. Axes represent normal modes; e.g., the long axis could be a bend and the short axis a higher frequency stretch with frequencies the same in both states.

potential energy surfaces is taken along a normal mode, the displacement is the distance between the bottom of the resulting parabolas. The reorganization energy is the increase in potential energy that results when one state is distorted to the geometry of the other state. The important parameters for electron transfer are the dimensionless displacements and reorganization energies, which depend on the frequency of the modes as well as the normal mode displacements. ${ }^{39} \mathrm{With}$ no coordinate rotation, each mode has its own reorganization energy, $\lambda_{\mathrm{i}}=0.5 v \Delta^{2}$, and the total vibrational reorganization energy is the sum of the contributions of each mode. ${ }^{40}$ In this simple case, the vector displacements to bring the coordinates in conjunction do not depend on the starting state since the vectors are equal in magnitude but opposite in sign. Starting with either state, this results in identical reorganization energies if the frequencies are the same, as is assumed here.

When the Duschinsky effect is taken into account, there is a relative rotation of the coordinate system in addition to a displacement. The Duschinsky effect is similar to two Cartesian coordinate systems rotated and displaced with respect to each other, with the normal modes as the axes (see Figure 2, middle and bottom drawings). When a one-dimensional slice of the potential energy surface is taken along a normal mode of one state, the slice does not correspond to a normal mode of the other state but goes along a linear combination of normal modes of the second state. The distance between the minima of the two surfaces, measured as the square root of the sum of squares of the normal mode displacements, is independent of which state is considered as the starting state. However, the simple interpretation of reorganization energy for the top drawing of Figure 2 does not apply to the two cases represented by the middle and bottom drawings. In our work, the reorganization energy parameters of Table 1 are based on the top drawing, a conventional and unambiguous definition. 
We now use Figure 2 to demonstrate how different types of coordinate rotations create a new perspective on reorganization energy. We will consider all drawings as having the upper state as the left set of contours. The middle drawing has the lower state rotated 20 degrees $\mathrm{CW}$ from the top drawing. If we consider a vertical transition from the excited state minimum (coordinate origin) to a point on the ground state coordinate system, we see that the energy in each mode on the ground state is not the same as in the top drawing. The middle drawing also shows that in absorption from the ground state to the excited state we reach a point on the upper surface that is the same as in the top drawing; this implies a constant reorganization energy in excited state coordinates. For the coordinate rotation in the middle drawing, we have a case where absorption will not be greatly affected by the amount of coordinate rotation, but in emission or ET the ground-state reorganization energy will be quite dependent on coordinate rotation. The bottom drawing is a different case, where we selected a CCW 20 degree rotation of the excited-state coordinate system. If we consider emission or ET from the excited state, it will reach the same point on the ground state as in the top figure. This case then has the groundstate reorganization energy independent of the degree of coordinate rotation. We note that absorption would reach a point on the upper surface that would be quite dependent on the degree of coordinate rotation but the emission would be less sensitive; this implies that the excited-state reorganization energy is dependent on the coordinate rotation.

In summary, the simple drawings of Figure 2 show how specific values of Duschinsky rotation could show a wide range of behavior for absorption and emission spectra as well as for plots of ET rates versus energy gap. In addition, the traditional definition of the reorganization energy is no longer applicable when there is a large Duschinsky effect. In general, even for modest Duschinsky rotation in a complex molecule, two reorganization energies can be computed where the value depends on which electronic state is used as a basis. As a result, features such as spectral peaks and rate maxima versus exothermicity no longer depend on the reorganization energy in the simple way normally assumed by ignoring Duschinsky effects. This is an important outcome that has previously been ignored in linking experiments and theory. Therefore, the validity of traditional ideas about reorganization energies depends on the degree of Duschinsky mixing. There is no simple redefinition of reorganization energy in the case of a large Duschinsky effect. For example, both in spectral peak locations and the location of rate maxima versus exothermicity, which is often used to assign reorganization energy, there often appears to be an effective reorganization energy that is different from either calculated value. We will give additional results in later sections to demonstrate these ideas.

Absorption and Emission Spectra for Case 1. In Figure 3, the absorption and emission spectra corresponding to Figure 1 are shown. It can be seen that the absorption spectrum changes little up to a mixing angle of about $30^{\circ}$. The rate, however, undergoes an increase of several orders of magnitude in this same range when deep in the inverted region. This is a little unexpected, and it would have been reasonable to assume that a large rate change should correspond with a significant change in the absorption spectrum. However, from the last section we anticipated this result, which is essentially based on the Condon principle. ${ }^{41}$ Since the Condon principle states that absorption is a vertical transition, the transition should be identical from the bottom of the ground state surface, which does not move as the surface is rotated, to the point on the excited state surface
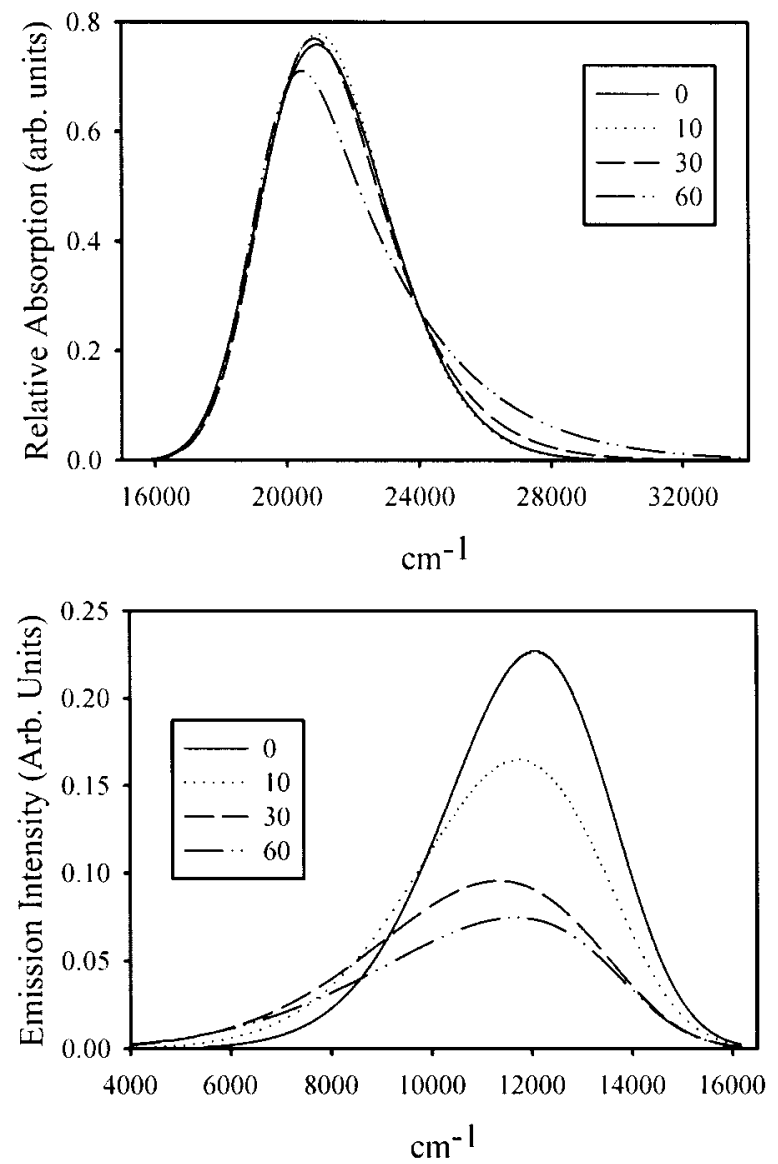

Figure 3. Calculated absorption and emission spectra corresponding to Figure 1 for $E_{00}=16000 \mathrm{~cm}^{-1}$.

directly above the bottom of the ground-state surface. Since the excited state is not rotated, the transition is to the same point on the excited-state surface (see top and middle drawings of Figure 2). However, wave functions do have width, and the spectral changes are due to the rotation of the ground-state wave function along with the coordinate system. This rotation changes the shape of the initial wave function with respect to the excitedstate surface and results in different Franck-Condon factors and slightly different absorption spectra for different amounts of coordinate rotation.

The emission spectrum in Figure 3 undergoes a great change when mixing is introduced. This is an example of an asymmetry between absorption and emission that occurs because of the Duschinsky effect. Normally, in the absence of the Duschinsky effect, the corrected absorption and emission spectra are mirror images of each other. However, the Duschinsky effect breaks this symmetry, as can be seen in Figure 3. For no mixing the spectra are roughly mirror images. However, as mixing is introduced in this case, the absorption spectrum remains largely unchanged, whereas the emission spectrum changes drastically. This can also be explained using the Condon principle. Now the initial state is the excited state, and, since the ground state is rotated, the mixing does not affect the excited state. However, the final state is rotated, meaning that the vertical transition now occurs to a different region of the potential energy surface (see top and middle drawing of Figure 2). Large changes in emission spectra are then expected as the coordinate rotates. The effects on the spectra are reduced when the frequency difference between the mixed modes is greater, which can make detection of Duschinsky effects from spectra more difficult.

The fundamental reason for the observed inverted region rate increase can be seen in the calculated low-temperature emission 


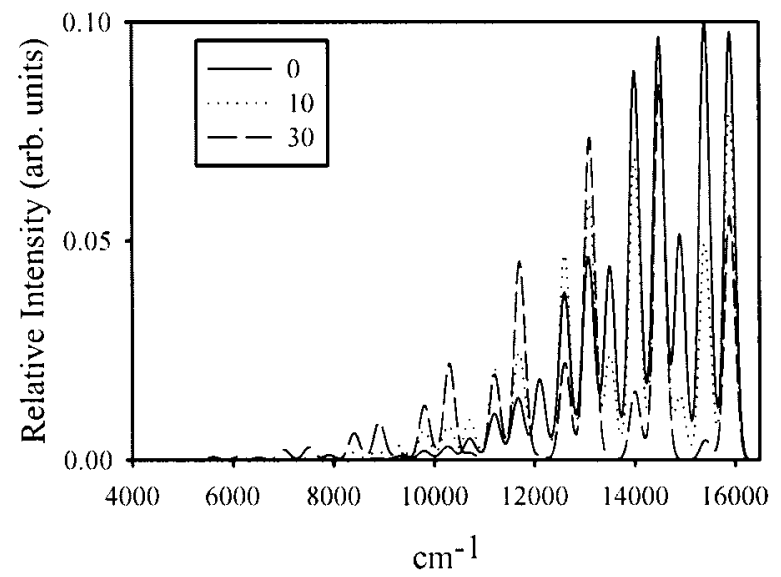

Figure 4. Calculated low-temperature emission spectra corresponding to Figure 1 for $E_{00}=16000 \mathrm{~cm}^{-1}, T=78^{\circ} \mathrm{K}$, and $\lambda_{\mathrm{s}}=100 \mathrm{~cm}^{-1}$.

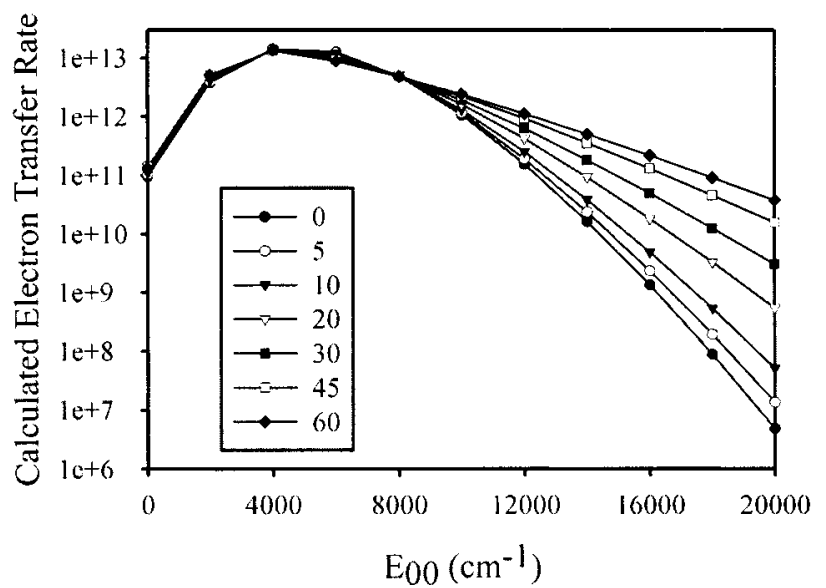

Figure 5. Calculated electron transfer rate vs energy gap for several values of mixing angle in Case 2, with a rotation of excited state normal coordinates. Parameters are the same as Figure 1 . Only the $500 \mathrm{~cm}^{-1}$ and $1400 \mathrm{~cm}^{-1}$ modes undergo the Duschinsky effect while also serving to accept $97 \%$ of the reorganization energy.

spectra shown in Figure 4. The low-temperature emission spectra are a good measure of the value of FCF between the lowest excited vibrational state and the various levels of the groundstate that are important in rate processes. As Figure 4 shows, as the amount of mixing increases, the lower energy emission bands increase in intensity. Low-energy emission corresponds to high-energy ground-state vibrational levels that are more important in inverted electron-transfer rates because of the Gaussian energy matching term in eq 2. Large relative effects are possible because of the extremely small value of these FCF in the no mixing case. Decreases in the high-energy emission intensity are also seen, consistent with the sum rule for FCF, but the relative changes are smaller. This helps to explain the small decrease in normal region ET rate with increased mixing.

Duschinsky Mixed Modes for Case 2, Rotation of Excited State. Examples of rate versus energy gap and absorption and emission spectra for rotation of the excited state coordinates are given in Figure 5 and Figure 6. As discussed in a prior section, this is a case based on the lower drawing of Figure 2. One difference between Figure 5 and Figure 1 is that the location of the maximum in the rate no longer moves with increased mixing. Also, the rate increase seen in the inverted region appears to be slightly smaller than in the previous case, although the general trend is the same. Also, there is less flattening of the rate versus energy gap for larger mixing. The changes in the absorption spectra are much more drastic in this case of excited state rotation. The location of the absorption maximum
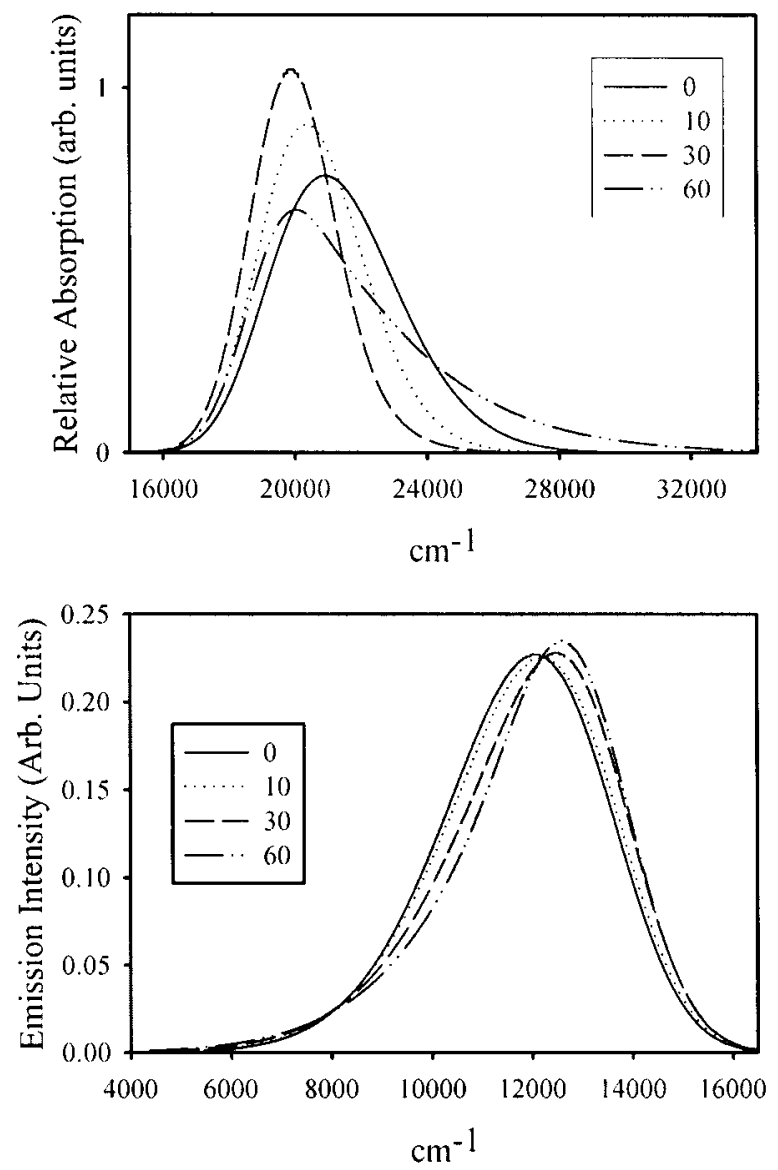

Figure 6. Calculated absorption and emission spectra corresponding to Figure 5 for $E_{00}=16000 \mathrm{~cm}^{-1}$.

and spectral width change a great deal as the mixing is increased, in contrast to what was seen for Case 1 . However, very little change is seen in the emission spectrum. This result can similarly be rationalized using the Condon principle (see top and bottom drawing of Figure 2). Smaller rate effects should be expected since only the shape of the initial wave function changes and not the final potential surface. In this case, the reorganization energy remains constant when referred to in ground-state coordinates, and the maximum in the rate versus energy gap does not move.

In both Case 1 and Case 2, the Duschinsky mixing caused significant rate constant effects, but the effects on spectra and rate versus energy gap depend on the details of the mixing. For a real molecule there is only one correct answer, which can be derived from exact computations of normal coordinates by ab initio methods when such methods are possible. From the experimental view, one often has either a featureless absorption or emission spectrum, which may not clearly identify the Duschinsky effect unless there is a dominant displaced oscillator that is also mixed. As we shall show in later sections, major rate effects can occur when mixed modes are only part of the reorganization energy and nontotally symmetric modes are surprisingly effective. If both absorption and emission are observable between the two states of interest, the amount of Duschinsky mixing can be approximated for displaced modes but not nontotally symmetric modes. In the case of no mixing, the absorption and emission spectra should be mirror images of each other after correcting for the frequency prefactors ( $v$ for absorption, $v^{3}$ for emission). A large asymmetry suggests a large Duschinsky effect.

B. Totally Symmetric Modes-Parameter Variations. Now that we have demonstrated that the Duschinsky effect can have 

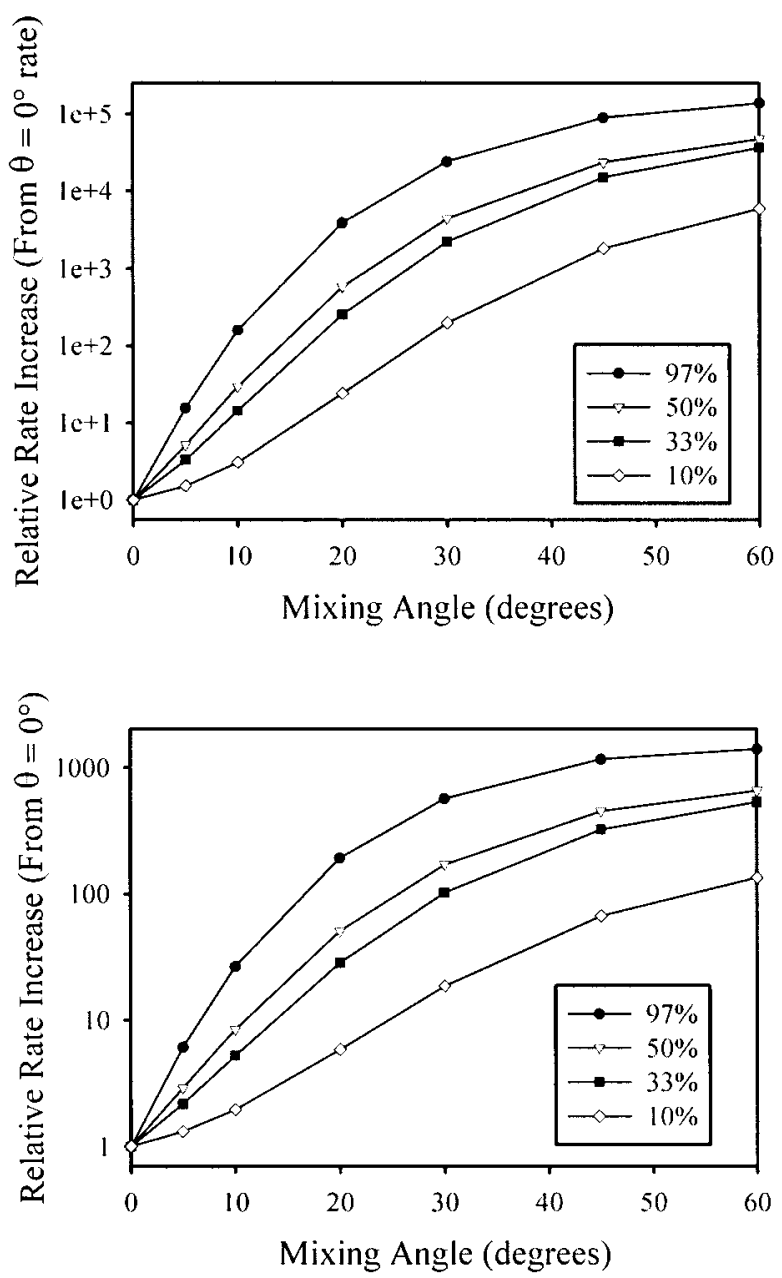

Figure 7. Relative rate increases at $E_{00}=20000 \mathrm{~cm}^{-1}$ (top) and 16000 $\mathrm{cm}^{-1}$ (bottom) as a function of mixing angle for different percentages of vibrational reorganization energy in the mixed $500 \mathrm{~cm}^{-1}$ and 1400 $\mathrm{cm}^{-1}$ modes. The reorganization energy in the $1200 \mathrm{~cm}^{-1}$ mode is increased to keep the total constant, and the relative amounts of reorganization energy in each mixed mode is fixed as in Figure 1.

important effects on ET rates, we will investigate more parameter changes and identify when rate effects are the largest. We have already shown that effects are large for ET in the inverted region of large energy gap. We will investigate some other issues to help identify under what conditions the Duschinsky effect is most important for ET rates. In the following studies, the ground state normal coordinate system will be rotated, as in Figure 1 and Figure 2 (middle drawing). Since the mixed modes in Figure 1 make up the dominant portion of the vibrational reorganization energy (97\%), we now test decreasing this percentage to more closely approximate typical molecules. We decreased the reorganization energy of the mixed modes $\left(1400 \mathrm{~cm}^{-1}, 500 \mathrm{~cm}^{-1}\right)$ and increased the reorganization energy of the $1200 \mathrm{~cm}^{-1}$ mode to keep the total constant, otherwise the parameters of Table 1 are the same. In Figure 7, the relative rate vs mixing angle at an energy gap of 20000 $\mathrm{cm}^{-1}$ (top figure) and $16000 \mathrm{~cm}^{-1}$ (bottom figure) is shown for several of these cases. Figure 7 shows that the larger the percentage of reorganization energy in the mixed modes, the larger the observed increase in rate. However, a large percentage is not needed since the rate increase is diminished only slightly in the case where the mixed modes are only $50 \%$ of the total vibrational reorganization. Even when the mixed modes account for only 10-20\% of the reorganization energy, the rate increase is still significant, showing that the mixed modes do not have

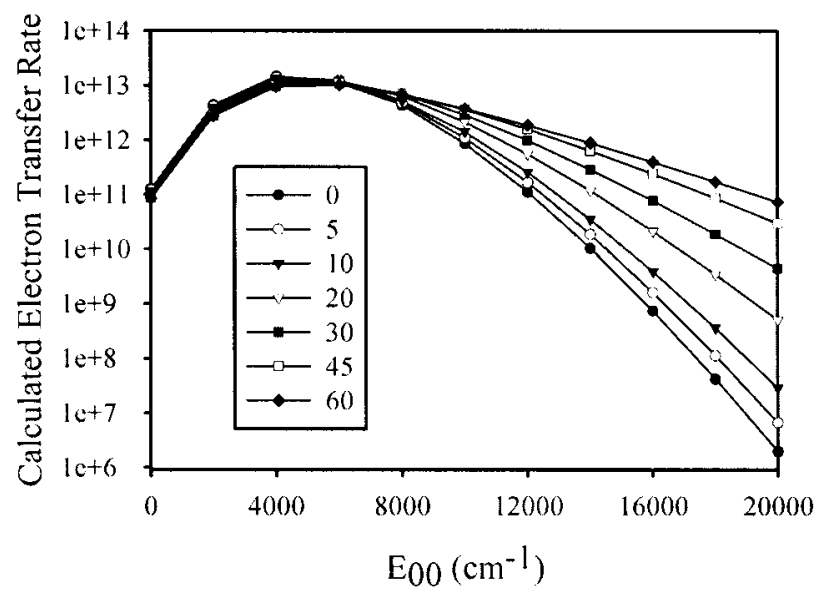

Figure 8. Calculated electron transfer rate vs energy gap for several values of mixing angle in Case 1, with a rotation of ground state normal coordinates. Parameters are given in Table 1, and only the $500 \mathrm{~cm}^{-1}$ and $1400 \mathrm{~cm}^{-1}$ modes undergo the Duschinsky effect while also serving to accept $33 \%$ of the reorganization energy with the $1200 \mathrm{~cm}^{-1}$ mode increased to provide the same total as in Figure 1.

to be the dominant modes for the Duschinsky effect to be important for ET rates. The magnitude of the rate effect depends on the energy gap; the effect is less at $16000 \mathrm{~cm}^{-1}$ and would decrease for smaller gaps but increase if the mixed modes are a large part of the reorganization energy.

The reduction of the mixed mode contribution to total reorganization energy has an effect on a plot of ET rate versus energy gap. If we reduce the contribution of the mixed modes to $33 \%$ of total reorganization energy instead of $97 \%$ in Figure 1, we create the plot of Figure 8, with otherwise similar parameters. We see from comparing these figures that the maximum of the curves shifts less in Figure 8 as the percent of mixing increases, and there is less flattening of the parabola for the highest degree of mixing. In real molecules it would be unusual, but possible, to have $97 \%$ of the acceptor modes also be Duschinsky mixed. Figures 1 and 8 both show that the maximum in the curve depends on the degree of mixing for Case 1. Since the curves in Figure 8 could be considered as common, there is a need to interpret the maximum as a sum of solvent and effective vibrational reorganization energy, which could deviate significantly from any simple computation of displaced modes without Duschinsky mixing. In addition, the significant flattening of the parabolas at the larger mixing values provides a new interpretation that might be applicable to experiments showing such a flattening of rate with energy gap. ${ }^{42,43}$

Figure 9 shows the electron-transfer rate as a function of mixing angle for $E_{00}=20000 \mathrm{~cm}^{-1}$ (top figure) and 16000 $\mathrm{cm}^{-1}$ (bottom figure) with different frequency ratios and reorganization energy ratios of the mixed modes. In all of these cases the total reorganization energy and the amount in the mixed modes (97\%) was kept constant and the same as in Figure 1 . We changed the value of the high frequency and the relative amount of displacement in the modes. For example, Figure 1 used the values 500:1400, 1:2.8 in the legend, which indicates that the reorganization energy was 2.8 times greater in the 1400 $\mathrm{cm}^{-1}$ mode than in the $500 \mathrm{~cm}^{-1}$ mode. If we contrast that result with the 500:1400, 1:1 plot we see an order of magnitude increase in rate when more of the reorganization energy (larger displacement) is in the low-frequency mode, and additional rate increases for the 500:1400, 2:1 case. We conclude that the larger the portion of reorganization energy that resides in the lower frequency mode, the larger the increase in rate. Also, it appears 

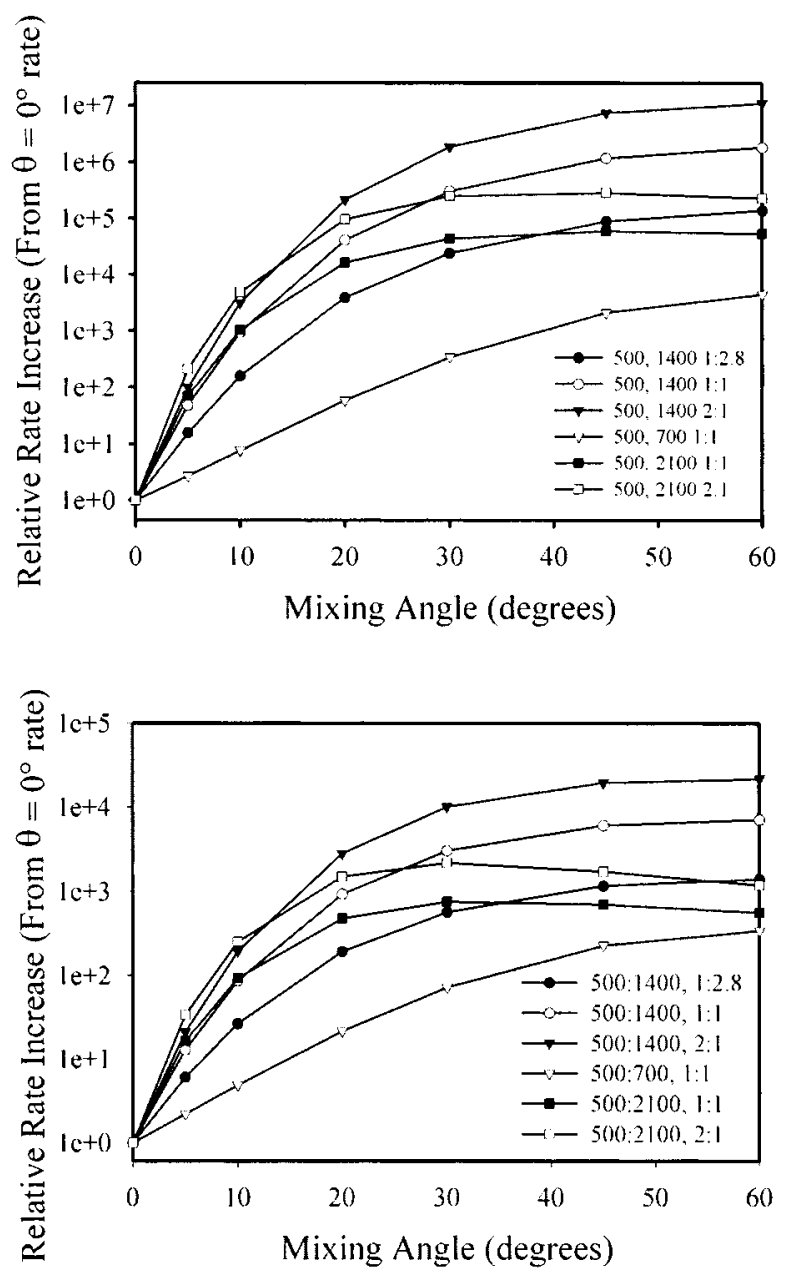

Figure 9. Relative rate increases at $E_{00}=20000 \mathrm{~cm}^{-1}$ (top) and 16000 $\mathrm{cm}^{-1}$ (bottom) as a function of mixing angle for different ratios of mixed mode frequencies and relative reorganization energy in the mixed modes. Ratios are listed as $v_{1}: v_{2}, \lambda_{1}: \lambda_{2}$, with total reorganization energy and the amount in the two mixed modes kept constant.

that for larger frequency differences in the mixed modes, there is a larger rate increase for a constant ratio of reorganization energies. For example, this suggests that a large rate effect may occur when the amount of bending and stretching character of a normal mode changes between electronic states and leads to mixing. However, for some parameters we are approaching an absolute rate that is quite large, so that there are significant plateau effects at larger mixing angles for the 500:2100 cases. This plateau can be best seen when comparing 500:1400, 2:1 and 500:2100, 2:1; however, for other parameters with smaller absolute rates, one finds that the trend holds and there are greater relative rate increases when there are greater frequency differences in the mixed modes.

In all of the examples given so far, the initial and final state frequencies have been the same. This has been done for simplicity, although it is not likely to be true in a real case. To test the validity of this assumption, changes were made to both initial and final state frequencies. Moderate frequency changes were seen to have a fairly small effect on the relative rate increase, sometimes increasing and sometimes decreasing the effect (not shown). It must be noted that this is true only for moderate changes in frequency. For extreme frequency changes (experimentally uncommon) the rate effects due to the frequency changes can be as important as Duschinsky mixing

The solvent is treated as a single classical harmonic oscillator, and therefore cannot undergo a Duschinsky effect in the current

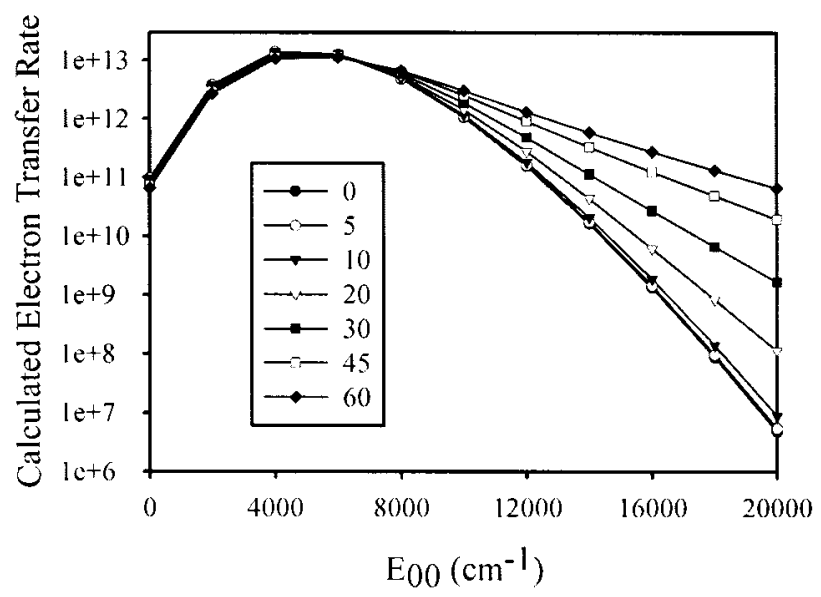

Figure 10. Calculated electron transfer rate vs energy gap for several values of mixing nontotally symmetric modes. Parameters are the same as Figure 1, but with the addition of $400 \mathrm{~cm}^{-1}$ and $1800 \mathrm{~cm}^{-1}$ modes having no displacement. Only the nontotally symmetric modes undergo the Duschinsky effect.

model. However the solvent reorganization energy still has a role in determining how Duschinsky mixing affects electrontransfer rates. The solvent reorganization energy, along with the vibrational reorganization energy, determines where inverted electron-transfer behavior begins, and therefore, when we can reach the inverted region of ET where the Duschinsky effect can become important. In addition to this classical effect, there is an additional effect that arises from the Gaussian energy matching term in eq 2 . The solvent reorganization energy and the temperature determine the width of this Gaussian, and therefore a larger solvent reorganization energy has a broader energy matching term that increases the number of FCF important in the rate calculation. In this case the important FCF now include lower ground-state quantum number states, which undergo smaller relative increases, or even decreases, with the inclusion of the Duschinsky effect. Large solvent reorganization energies should reduce the rate effects that arise from the Duschinsky effect.

\section{Nontotally Symmetric Modes Have Large Duschinsky} Effects

Nontotally symmetric modes are ignored in standard ET theories. This is due to a lack of Franck-Condon activity, since, in the absence of Duschinsky mixing or frequency changes, nontotally symmetric modes have no contribution to the rate since their FCF are zero. In addition, any contributions that arise from frequency changes are usually small and constitute a negligible contribution to the rate. However, we expected that Duschinsky mixing of nontotally symmetric modes could provide a significant rate contribution since mixing two nontotally symmetric modes that have a relatively large difference in frequency should be analogous to a large frequency change.

In Figure 10 a plot of rate vs energy gap for a system mixing 400 and $1800 \mathrm{~cm}^{-1}$ nontotally symmetric modes is shown. Results are similar to the totally symmetric mode case, with inverted region rates increasing with increased mixing. However, in this case the rate shows a maximum at an energy gap equal to the total reorganization energy, for all values of the mixing angle, unlike the totally symmetric mode cases. This is due to the fact that since nontotally symmetric modes have no displacement, they contribute nothing to the reorganization energy. This provides another insight into our assignment of an effective reorganization energy to the peak shifting in Figure 1 for the totally symmetric mode case. An additional difference with Figure 1 is the magnitude of the rate increase, especially 

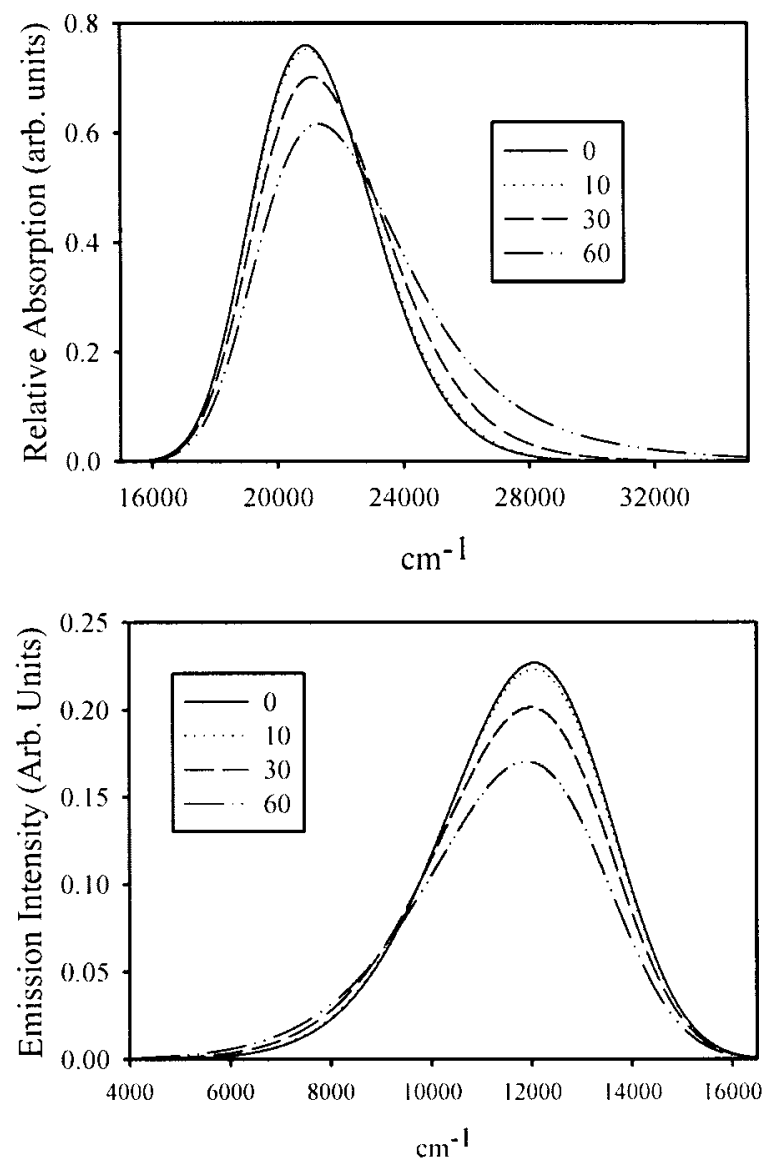

Figure 11. Calculated absorption and emission spectra for mixing of nontotally symmetric modes that corresponds to Figure 10 for $E_{00}=$ $16000 \mathrm{~cm}^{-1}$

at low mixing. A mixing angle of $10^{\circ}$ does very little to the rate in this instance, and the rate starts to increase significantly between $10^{\circ}$ and $20^{\circ}$. In the totally symmetric case the increase in rate began immediately, and a significant increase is already seen at $10^{\circ}$.

Figure 11 shows corresponding calculated absorption spectra for an energy gap of $16000 \mathrm{~cm}^{-1}$. Again, at low mixing very little change is observed. Very small rate changes are also seen at low mixing. However, a high energy tail then begins to appear at larger mixing, which causes significant broadening of the spectrum. These changes are much more pronounced than the absorption in Figure 3, but much less than absorption changes in Figure 6. A very similar effect is seen in the emission spectrum. If the spectra are corrected by dividing by the prefactors, the absorption and emission are mirror images, even in the case of large mixing. It must be noted that because there is no displacement along these nontotally symmetric modes, the results do not depend on which state is rotated; therefore, we do not observe the large differences between Case 1 and Case 2 of totally symmetric modes. When all of the mixing is in nontotally symmetric modes the rotation of the excited state is equivalent to rotating the ground state in the opposite direction. In addition, a negative rotation gives the same results as a positive rotation, since there is no displacement along these modes. ${ }^{18}$ The rate, absorption, and emission effects arise from the fact that the FCF with higher lying final vibrational states are increased when the Duschinsky effect is introduced.

Figure 12 shows the effect of changing the frequency of the mixed nontotally symmetric modes. The lower frequency is kept the same while the upper frequency is changed from 1800 to $800 \mathrm{~cm}^{-1}$ or $2800 \mathrm{~cm}^{-1}$. It appears that the greater the difference

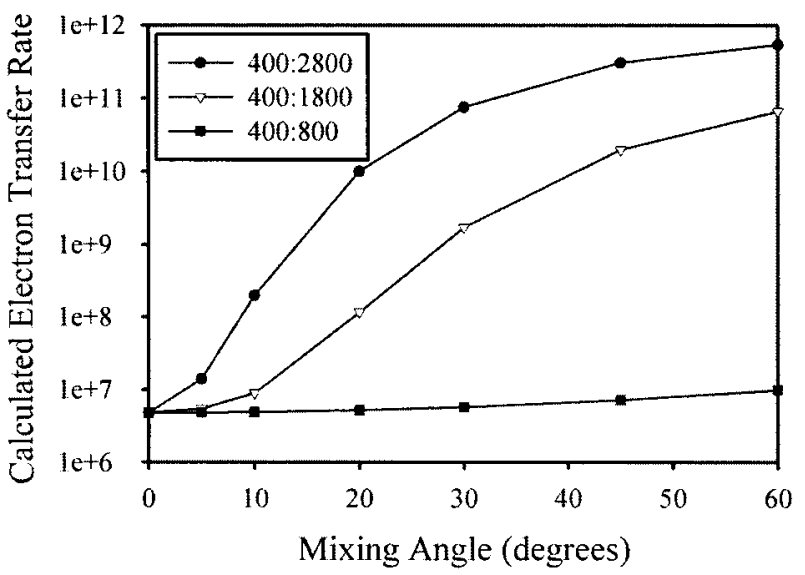

Figure 12. Calculated electron transfer rate as a function of mixing angle for several frequency ratios of Duschinsky mixed, nontotally symmetric modes.

in frequency the greater the rate effect. In fact for the $800 \mathrm{~cm}^{-1}$ case, there is essentially no rate increase. These results support assigning the rate effects to effective frequency changes that result from mixing modes of greatly differing frequencies. However, it should be noted that these calculated rate effects are smaller than seen in the totally symmetric cases mentioned previously. This result shows that Duschinsky mixing of displaced, totally symmetric modes has a larger effect than mixing nondisplaced, nontotally symmetric modes. The importance of mode displacement when mixing modes probably arises from the enhanced FCF that come from involving different portions of the potential energy surfaces.

The commonly held assumption that nontotally symmetric modes are of no importance is not correct, and we have shown that they can have a considerable effect on ET rates. In addition, for real molecules there can be a large additive effect of mixing many nontotally symmetric modes to the FCF component in the rate since generally many more such modes exist, particularly at low frequencies. Furthermore, nontotally symmetric modes of very low frequency might be more easily mixed with high frequencies of similar symmetry, although this issue can be judged only for specific molecules.

\section{Conclusions}

The Duschinsky effect has been shown to be a very important factor in calculating inverted region ET rates. When displaced, totally symmetric modes are involved, rates can increase several orders of magnitude for inverted electron transfer reactions. The peak location in a rate vs energy gap plot can depend on the degree of Duschinsky mixing, and therefore it corresponds to a sum of solvent and an effective vibrational reorganization energy that is not interpretable by simple models that exclude mixing. We demonstrate that large rate effects can occur with very little change in either the absorption or emission spectra, depending on the details of the Duschinsky mixing. These results imply that experiments without both absorption and emission spectra cannot determine if Duschinsky effects are present. Furthermore, the large rate effects for Duschinsky mixing of nontotally symmetric modes make the absorption and emission spectra useful only for displaced modes. The origin of the ET rate effect is the increased Franck-Condon factors between the initial vibrational state and the higher lying final vibrational states when the Duschinsky effect is taken into account. The rate effect of mixing two nontotally symmetric modes is smaller than the effect of two totally symmetric modes. However, they have large 
enough rate increases at moderate mixing to allow several nontotally symmetric vibrational modes to be as important as totally symmetric modes in determining ET rates. The effects of nontotally symmetric modes are more important when the frequencies are very different, and since there are many nontotally symmetric modes, there is ample opportunity for a large total contribution. Nontotally symmetric modes do not have the asymmetry between absorption and emission spectra as seen for displaced modes, and the peak in a rate versus energy gap can be interpreted conventionally since they do not contribute to reorganization energy.

Previously, the Duschinsky mixing of normal coordinate vibrations has not been systematically applied to computing transition rates for electron transfer or any other rate process. We have identified large ET rate effects from Duschinsky mixing, and find that both totally symmetric and nontotally symmetric modes are very important, unlike conventional models of ET. The inclusion of the Duschinsky effect will be crucial for predicting ET rates and explaining experimental rates of ET. Other experimental discrepancies require investigation; for example, for plots of ET rate versus increasing energy gap a flattening of the parabola in the high energy gap region can be clearly seen in some of our examples. In addition, the peak location in such a plot includes an effective vibrational reorganization energy that is not predictable without the Duschinsky effect.

Acknowledgment. We thank the U.S. Department of Energy, Office of Science (Grants DE-FG02-87ER13808 for J.T.H. and DE-FG02-91ER14228 for K.G.S.) for financial support.

\section{References and Notes}

(1) Bixon, M.; Jortner, J. In Electron Transfer-From Isolated Molecules to Biomolecules; Bixon, M., Jortner, J., Eds.; John Wiley \& Sons: New York, 1999; pp 35-202.

(2) Duschinsky, F. Acta Physicochim. URSS 1937, 7, 551.

(3) Ruhoff, P. T., Ph.D. Thesis, Odense University, 1995.

(4) Ozkan, I. J. Mol. Spec. 1990, 139, 147-162. 3463.

(5) Sharp, T. E.; Rosenstock, H. M. J. Chem. Phys. 1964, 41, 3453-

(6) Sando, G. M.; Spears, K. S. J. Phys. Chem. A. 2001, 105, 5326.

(7) Mebel, A. M.; Chen, Y. T.; Lin, S. H. Chem. Phys. Lett. 1996, $258,53-62$.

(8) Mebel, A. M.; Chen, Y. T.; Lin, S. H. J. Chem. Phys. 1996, 105, 9007-9020.

(9) Mebel, A. M.; Lin, S. H.; Chang, C. H. J. Chem. Phys. 1997, 106, $2612-2620$.
(10) Mebel, A. M.; Chen, Y. T.; Lin, S. H. Chem. Phys. Lett. 1997, $275,19-27$.

(11) Liao, D. W.; Mebel, A. M.; Hayashi, M.; Shiu, Y. J.; Chen, Y. T.; Lin, S. H. J. Chem. Phys. 1999, 111, 205-215.

(12) Hemley, R. J.; Lasaga, A. C.; Vaida, V.; Karplus, M. J. Chem. Phys. 1988, 92, 945-954.

(13) Clauberg, H.; Chen, P. J. Phys. Chem. 1992, 96, 5676-5678.

(14) Olbrich, G.; Kupka, H. Z. Naturforsch. 1983, 38a, 937-946.

(15) Hemley, R. J.; Dawson, J. I.; Vaida, V. J. Chem. Phys. 1983, 78, 2915-2927.

(16) Myers, A. B.; Pranata, K. S. J. Phys. Chem. 1989, 93, 5079-5087.

(17) Phillips, D. L.; Zgierski, M. Z.; Myers, A. B. J. Phys. Chem. 1993, 97, 1800-1809.

(18) Mebel, A. M.; Hayashi, M.; Liang, K. K.; Lin, S. H. J. Phys. Chem. A 1999, 103, 10674-10690.

(19) Hayashi, M.; Mebel, A. M.; Liang, K. K.; Lin, S. H. J. Chem. Phys. 1998, 108, 2044-2055.

(20) Lee, E.; Medvedev, E. S.; Stuchebrukhov, A. A. J. Chem. Phys. 2000, 112, 9015-9024.

(21) Lee, E.; Medvedev, E. S.; Stuchebrukhov, A. A. J. Phys. Chem. B 2000, 104, 6894-6902.

(22) Spears, K. G.; Shang, H. J. Phys. Chem. A 2000, 104, 2668-2680.

(23) Knochenmuss, R.; Muino, P. L.; Wickleder, C. J. Phys. Chem. 1996, $100,11218-11227$.

(24) Mochizuki, Y.; Kaya, K.; Ito, M. Chem. Phys. 1981, 54, 375382.

(25) Venuti, E.; Marconi, G. J. Mol. Struct. 1992, 266, 235-239.

(26) Wunsch, L.; Metz, F.; Neusser, H. J.; Schlag, E. W. J. Chem. Phys. 1977, 66, 386-400.

(27) Hougen, J. T.; Watson, J. K. G. Can. J. Phys. 1965, 43, 298-320.

(28) Ruhoff, R. T. Chem. Phys. 1994, 186, 355-374.

(29) Islampour, R.; Dehestani, M.; Lin, S. H. J. Mol. Spectrosc. 1999, 194, 179-184.

(30) Chen, K.; Pei, C. Chem. Phys. Lett. 1990, 165, 523-527.

(31) Faulkner, T. R.; Richardson, F. S. J. Chem. Phys. 1979, 70, $1201-$ 1213.

(32) Kupka, H.; Cribb, P. H. J. Chem. Phys. 1986, 85, 1303-1315.

(33) Ruhoff, P. T.; Ratner, M. A. Int. J. Quantum Chem. 2000, 77, 383392.

(34) Myers, A. B. Chem. Phys. 1994, 180, 215-230.

(35) Bolton, J. R.; Archer, M. D. Adv. Chem. 1991, 228, 7-23.

(36) Yan, Y. J.; Mukamel, S. J. Chem. Phys. 1986, 85, 5908-5923.

(37) Wynne, K.; Galli, C.; Hochstrasser, R. M. J. Chem. Phys. 1994, $100,4797-4810$

(38) Todd, M. D.; Nitzan, A.; Ratner, M. A.; Hupp, J. T. J. Photochem. Photobiol. A: Chem. 1994, 82, 87-101.

(39) Myers, A. B. In Laser Techniques in Chemistry; Myers, A. B., Rizzo, T. R., Eds.; John Wiley \& Sons: New York, 1995; pp 325-384.

(40) Doorn, S. K.; Hupp, J. T. J. Am. Chem. Soc. 1989, 111, 11421144 .

(41) Condon, E. U. Phys. Rev. 1928, 32, 858.

(42) Asahi, A.; Mataga, N. J. Phys. Chem. 1989, 93, 6575-6578.

(43) Wasielewski, M. R.; Gaines, G. L.; O’Neil, M. P.; Svec, W. A.; Niemczyk, M. P.; Prodi, L.; Gosztola, D. In Dynamics and Mechanism of Photoinduced Transfer and Related Phenomena; Mataga, N., Okada, T., Masuhara, H., Eds.; Elsevier Science Publishers: New York, 1992; pp $8-21$. 